\title{
Simulation of the Retroglossal Fluid-Structure Interaction During Obstructive Sleep Apnea
}

\author{
Franz Chouly ${ }^{1,2}$, Annemie Van Hirtum² ${ }^{2}$, Pierre-Yves Lagrée ${ }^{3}$, Jean-Roch \\ Paoli $^{4}$, Xavier Pelorson ${ }^{2}$, and Yohan Payan ${ }^{1}$ \\ 1 Laboratoire TIMC, UMR CNRS 5525, Université Joseph Fourier, 38706 La \\ Tronche Cedex, France. \{Franz.Chouly, Yohan.Payan\}@imag.fr \\ 2 Institut de la Communication Parlée, INPG, UMR CNRS Q5009, 38031 Grenoble \\ Cedex, France. \{annemie,pelorson\}@icp.inpg.fr \\ 3 Laboratoire de Modélisation en Mécanique, UMR CNRS 7607, B 162, Université \\ Paris 6, 75252 Paris, France. pyl@ccr.jussieu.fr \\ 4 Centre Hospitalier Universitaire Purpan, Place du Docteur Baylac, TSA 40031, \\ 31059 Toulouse Cedex 9, France. paoli.jr@chu-toulouse.fr
}

\begin{abstract}
A method for computing the interaction between the airflow and the soft tissue during an Obstructive Apnea is presented. It is based on simplifications of the full continuum formulation (NavierStokes and finite elasticity) to ensure computation time compatible with clinical applications. Linear elasticity combined with a precomputation method allows fast prediction of the tissue deformation, while an asymptotic formulation of the full Navier-Stokes equations (Reduced NavierStokes/Prandtl equations) has been chosen for the flow. The accuracy of the method has already been assessed experimentally. Then, simulations of the complete collapsus at the retroglossal level in the upper airway have been carried out, on geometries extracted from pre-operative radiographies of two apneic patients. Post-operative geometries have been also used to check qualitatively if the predictions from the simulations are in agreement with the effects of the surgery.
\end{abstract}

\section{Introduction}

The Obstructive Sleep Apnea Syndrome is now identified as a major health care topic, which affects a growing part of the population in the western countries [1]. At least, $2 \%$ of the women and $4 \%$ of the men suffer from this disorder [2]. Its main consequences are excessive daytime sleepiness and an increased risk of cardiovascular diseases [1]. Fundamentally, it is caused by the interaction between the respiratory airflow and the soft tissue in the pharynx [3]. Physical modelling of this complex phenomenon is of interest both for going deeper into the understanding of its mechanisms and for further improvements of the treatments. In particular, realistic numerical simulations of the interaction between the airflow and the pharyngeal structures during Obstructive Sleep Apnea, on geometries obtained from medical imaging, might be helpful for the clinician or the surgeon, who attempt at modifying the geometry or the mechanical properties of the upper airway. In this perspective, two prerequisites should be considered. The first 
is that the simulations should be validated experimentally, so that the clinician may trust their predictions. The second is that computational cost in a clinical context should be low. Indeed, for this problem, the best method would be to solve accurately the full unsteady Navier-Stokes equations in interaction with soft tissue governed by non-linear viscoelastic behaviour, in large deformations, using tridimensional data such as CT-scans or MRI. However, this complete formulation is very difficult to solve numerically (convergence problems on real in-vivo geometries) and requieres hours if not days of computations [4] [5]. A reasonable objective for clinical applications should be a simulation time of less than one hour, or even of a few minutes, so that many simulations, involving different modifications of the morphology or of the tissue properties, should be carried out one after the other. Therefore, the objective of this paper is to present numerical simulations that meet these two prerequisites, which has never been done before for Obstructive Sleep Apnea ${ }^{5}$. The method, as well as the framework for simulations, is first explained in section 2. In particular, the simplifications in the assumptions and in the numerical solving, in view of the second prerequisite, are given. For the first prerequisite, systematic comparison to in-vitro measurements has already been proceeded and is detailed in [8] [9] [10], so we focus here on simulations from in-vivo data. Therefore, in section 3 , the simulation of an apnea is detailed, followed by confrontation between simulations from preoperative and post-operative data, which will provide a preliminary qualitative in-vivo evaluation of the method.

\section{Material and Methods}

The construction of a patient geometry from a radiography is first described, followed by the method of computation for simulations. Finally, the choice of values for physical parameters and the estimation of the reference configuration after the application of gravity are detailed.

\subsection{Extraction of the Geometry from Sagittal Radiographies}

The upper airway geometry of two apneic patients was obtained from sagittal radiographies. These two patients undergone maxillomandibular surgery: their maxilla and mandible were translated forward in the antero-posterior direction, of approximatively 8 to $10 \mathrm{~mm}$. Two acquisitions took place for each of them: one before and one after the surgical intervention, at approximatively one month of interval. The main interest of the radiographies is to dispose of the post-operative data, since in the normal surgical protocol, no MRI or CT-scans are taken after

\footnotetext{
${ }^{5}$ Most of the simulations for biomechanical processes at the macroscopic level involving fluid-structure interaction are devoted to blood flows or to the pulmonary airway. To our knowledge, the only published numerical simulations for Obstructive Sleep Apnea are those of B. Shome and coll. [6], focused on the fluid flow (no interaction with the soft tissue), and of A. Malhotra and coll., in which computation time is not indicated and which have not been validated experimentally [7]
} 


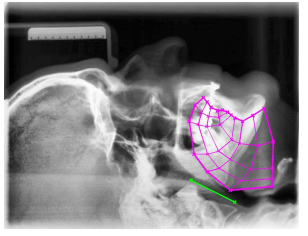

(PreOp)

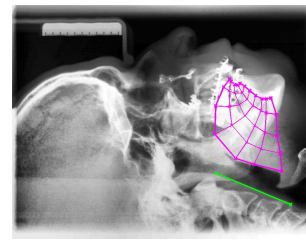

(PostOp)

Fig. 1. Segmentation of the pre-operative ( $\mathrm{PreOp}$ ) and the post-operative (PostOp) radiographies of apneic patient no. 1. The initial mesh and the borders of the tongue are displayed in purple, while the posterior pharyngeal wall is in green

the intervention. Table 1 provides patients clinical information. In particular, the Apnea-Hypopnea Index (AHI) drops dramatically after the intervention, which shows the efficiency of the surgical gesture.

From each radiography, a bidimensional model of the tongue has been extracted. First, landmarks have been manually positioned in order to determine the border of the tongue. From these landmarks, a mesh has been generated. It is a structured, isoparametric mesh, as in [11], made of 216 quadrilateral linear elements. The posterior pharyngeal wall has been considered in a first approximation as a straight segment. The airway is naturally delimited by the base of the tongue and the posterior pharyngeal wall. An example of segmentation is depicted in figure 1. For the two patients considered in this paper, segmentations have been validated by a clinical expert.

\subsection{Computation of the Fluid-Structure Interaction}

As pointed out in the introduction, since our objective is a low computational cost, some simplifications in the formulation of the problem have been adopted. First of all, in agreement with the clinical data at our disposal, a bidimensional formulation has been chosen, both for the soft tissue and for the airflow. Moreover, the problem is considered as quasi-steady, since the duration of the closure lasts about a few seconds. This allows to solve the fluid-structure interaction using a segregative method: the equations that govern the fluid and the structure are solved alternatively [12]. For the soft tissue, small deformations and small displacements assumptions have been thought as suitable. This is different from a context such as speech production where large deformations are involved [13]. The material has been chosen as homogeneous, isotropic and linear (Hookean material). The resulting equations are solved using the finite element method [14]. Within this framework, the relationship between the nodal displacements $\{\mathbf{u}\}$ and the nodal forces $\{\mathbf{F}\}$ is linear:

$$
[\mathbf{K}]\{\mathbf{u}\}=\{\mathbf{F}\},
$$

where $[\mathbf{K}]$ is the stiffness matrix [14]. Then, a method of precomputation, similar to the one detailed in [15], has been used. It consists in computing the invert 
of $[\mathbf{K}]$ before entering into the fluid-structure interaction loop. Ansys(TM) software was used at this effect. As the displacement at each node is simply obtained from a matrix multiplication at each step of the fluid-structure interaction loop, the precomputation of the invert of $[\mathbf{K}]$ saves a great amount of computation time. The airflow has been considered as :

- incompressible : the Mach number is of the order of $10^{-2}[8]$,

- laminar : the Reynolds number is of the order of $10^{3}[8]$. We neglected the turbulent effects due to shear instability in the jet that appears downstream the base of the tongue. In fact, the flow may be transitional, but, as shown in the experimental study [8], laminar regime remains a satisfying first approximation.

- stationnary: the Strouhal number is of the order of $10^{-3}$ [8]. Moreover, the displacement speed of the soft tissue is very slow in comparison to the characteristic time of the flow, so that unstationnary effects should be neglected in the fluid ${ }^{6}$.

Working at Reynolds number of the order of $10^{3}$ allows to use an asymptotical simplification of the incompressible Navier-Stokes equations:

$$
\begin{aligned}
\bar{u} \partial_{\bar{x}} \bar{u}+\bar{v} \partial_{\bar{y}} \bar{u} & =-\partial_{\bar{x}} \bar{p}+\partial_{\bar{y}^{2}}^{2} \bar{u}, \\
-\partial_{\bar{y}} \bar{p} & =0, \\
\partial_{\bar{x}} \bar{u}+\partial_{\bar{y}} \bar{v} & =0,
\end{aligned}
$$

where $(\bar{u}, \bar{v})$ are the longitudinal $(\bar{x})$ and transverse $(\bar{y})$ components of the fluid velocity, and $\bar{p}$ is the pressure [16]. All the variables are nondimensional: $\bar{x}=x\left(h_{0} R e\right)^{-1}, \bar{y}=y h_{0}^{-1}, \bar{p}=P\left(\rho U_{0}^{2}\right)^{-1}, \bar{u}=u U_{0}^{-1}, \bar{v}=v R e U_{0}^{-1} . h_{0}$ is the transversal dimension of the pharyngeal duct, $U_{0}$ is the mean longitudinal speed, and $R e$ is the Reynolds number $\left(R e=U_{0} h_{0} / \nu\right.$, with $\nu$ the kinematic viscosity of the air). This simplification, called Reduced Navier-Stokes / Prandtl (RNSP) equations, allows to take into account the boundary layer formation and the separation of the fluid after the narrowing of the pharyngeal duct, at the base of the tongue. Furthermore, it allows fast and efficient numerical solving, based on a finite difference scheme [16]. For more details about the assumptions, the mathematical formulation and the numerical solving, one can refer to [9] [10]. Finally, the method has been extensively validated thanks to comparison with pressure and deformation measurements on an in-vitro setup. For the computation of the fluid flow, the above assumptions and the RNSP equations have been validated on a rigid setup [8]. For the fluid-structure interaction, a deformable setup has been used [9] [10]. As a result, the first prerequisite is satisfied. For the second prerequisite, the duration of the computations is typically of the order of 20 minutes (see 4.1), which can be considered as correct for clinical applications.

$\overline{6}$ in opposition to applications such as snoring or speech production in which it would not be the case 


\subsection{Framework for the Simulations}

Table 1. Information on each patient, after pre-operative ('PreOp') and post-operative ('PostOp') examination. 'AHI' is the Apnea-Hypopnea Index, which is the number of obstructive events per hour of sleep, measured during polysomnography. $h_{c}^{i}$ is the constriction height in vertical position, awake, as it is measured from the sagittal radiographies. $h_{c}^{f}$ is the constriction height in a sleep supine position, after the application of the gravity loads

\begin{tabular}{|l||l|l||l|l|}
\hline Patient & 1 & & 2 & \\
\hline Examination & PreOp & PostOp & PreOp & PostOp \\
\hline \hline AHI & 80 & 8 & 49 & 0 \\
\hline$h_{c}^{i}(\mathrm{~mm})$ & 5.9 & 14.1 & 6.6 & 16.8 \\
\hline$h_{c}^{f}(\mathrm{~mm})$ & 1.5 & 10.5 & 1.8 & 12.9 \\
\hline
\end{tabular}

For each bidimensional model, the plane strain assumption has been chosen. The depth ${ }^{7}$ has been fixed to $30 \mathrm{~mm}$. It corresponds to a mean value of the depth of the oropharyngeal airway. The Poisson's ratio has been fixed to 0.499. It stands for quasi-incompressibility, in agreement with the fact that the soft tissues are mostly made of water. The same value for the Young modulus has been used for all the simulations: $6 \mathrm{kPa}$. It corresponds to tissue in passive configuration [7]. The boundary conditions are the following: the tongue is attached to the mandibulae and to the hyoid bone, which have been considered as immobile, in agreement with remarks from the clinical expert. Then, before the simulations of the interaction with the airflow, the influence of the gravity has been simulated. Indeed, as the radiographies were taken in vertical position, during wakefulness, a new configuration corresponding to sleep in supine position has to be computed. The norm of the gravity field $\mathbf{g}$ has been fixed to $9.81 \mathrm{~m} . \mathrm{s}^{-2}$. The density $\rho$ of the tissue has been fixed to $1000 \mathrm{~kg} \cdot \mathrm{m}^{-3}$. The configuration obtained after the application of gravity loads has been considered as the reference configuration for all the computations described in the next section. Table 1 gives some information about the deformation induced by gravity, especially the minimal height of the duct, at the extremity of the base of the tongue. It has been called the constriction height $h_{c}$.

\section{$3 \quad$ Results}

Simulations have been carried out on the oropharynx models built from the sagittal radiographies, before and after surgery. Comparison of the tongue deformation in response to inspiratory airflow allows us to check if the predictions from the simulations are in agreement with the consequences of the surgical intervention. In order to generate an inspiratory pattern, the inlet pressure has

\footnotetext{
${ }^{7}$ or the dimension in the direction orthogonal to the sagittal plane
} 
been fixed to $0 \mathrm{~Pa}$ while the outlet pressure decreases from $0 \mathrm{~Pa}$ to a negative chosen value $P_{s} \mathrm{~Pa}$.

\subsection{Simulation of an Obstructive Apnea}

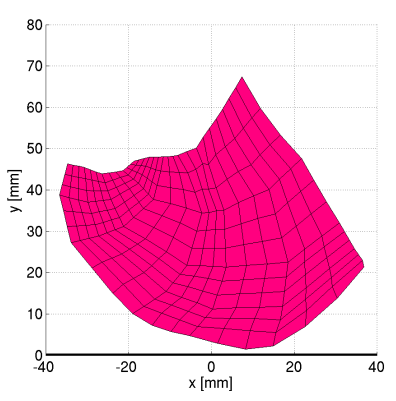

(a)

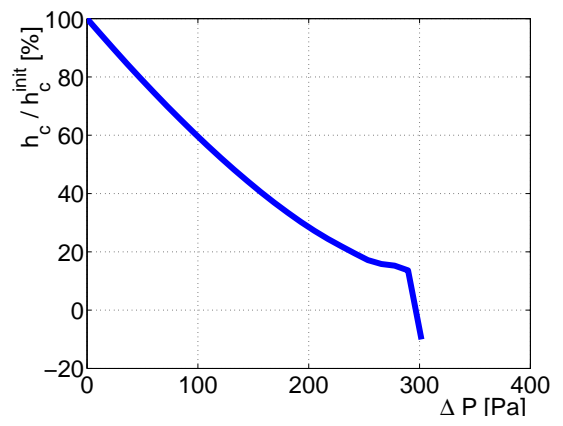

(c)

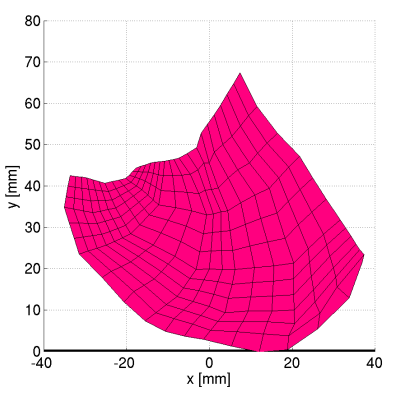

(b)

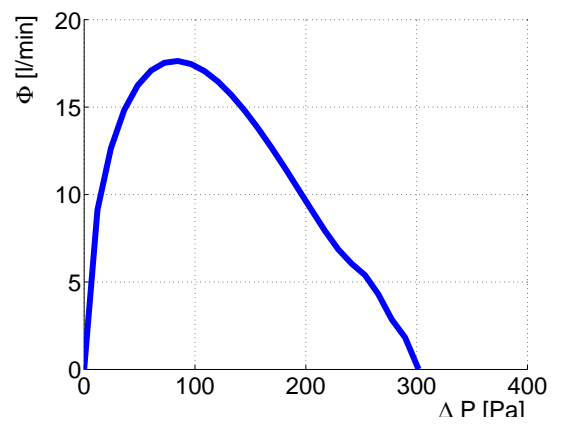

(d)

Fig. 2. Simulation of the upper airway obstruction from pre-operative data. (a) Initial configuration. (b) Final configuration. (c) Constriction height. (d) Airflow rate $\phi$

On figure 2 is depicted the simulation of a complete upper airway collapsus. The geometry is extracted from the pre-operative data of the patient no. 1 . The constriction height $h_{c}$ decreases from its initial value to $0 \mathrm{~mm}$ (figure (c)), at which collision with the posterior pharyngeal wall is detected (figure (b)). In parallel, the airflow rate $\phi$ increases with the pressure drop $\Delta P$ until approximatively $80 \mathrm{~Pa}$, where the maximal value of $17.5 \mathrm{l} / \mathrm{min}$ is reached (figure (d)). After $80 \mathrm{~Pa}$, the flow rate $\phi$ decreases as the pressure drop $\Delta P$ is increased. In this part of the curve, the relationship $\phi(\Delta P)$ is approximatively linear. This phenomenon of flow limitation is caused by the narrowing of the pharyngeal duct, which effect counterbalances the increasing pressure drop. It has been observed in clinical conditions during a hypopnea, which are caused by partial closure of the upper airway [17]. Finally, when the pressure drop $\Delta P$ reaches approximatively $300 \mathrm{~Pa}$, the flow stops as the airway is closed. This complete closure of the airway in response to inspiratory airflow corresponds to an obstructive apnea. 


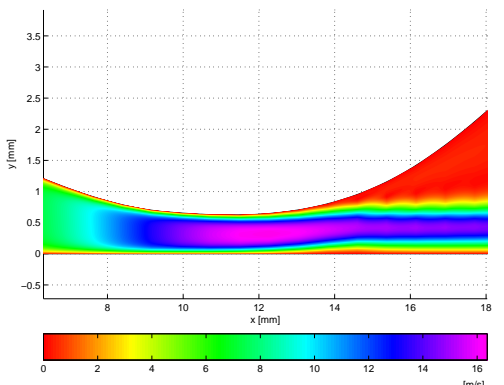

(a)

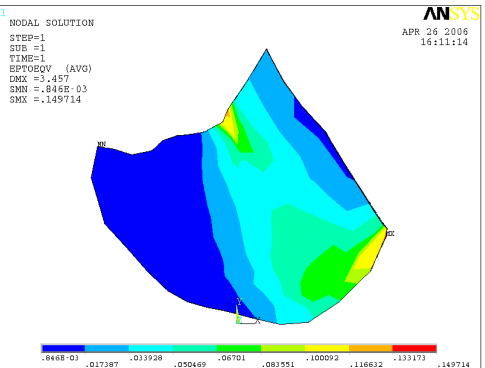

(b)

Fig. 3. Simulation of the upper airway obstruction from pre-operative data. (a) Velocity of the fluid at the level of the constriction (isovalues). (b) Von Mises strain

More details about the kinematics are given in figure 3. The figure 3 (a) depicts the flow velocity in the pharyngeal duct during the closure (when $\Delta P=$ $145 \mathrm{~Pa}$ and $\phi=14.8 \mathrm{l} / \mathrm{min}$ ). The phenomena already mentionned in 2.2 of flow separation and of jet formation can be observed. On the figure 3 (b), the Von Mises strain distribution at the end of the simulation, after complete closure, has been plotted. The mean value is of the order of $5 \%$, which confirms the small deformations assumption. The peak values, of less than $20 \%$, are at the level of the mandible and of the hyoid bone (fixed nodes).

\subsection{Comparison between Pre-Operative and Post-Operative Data}

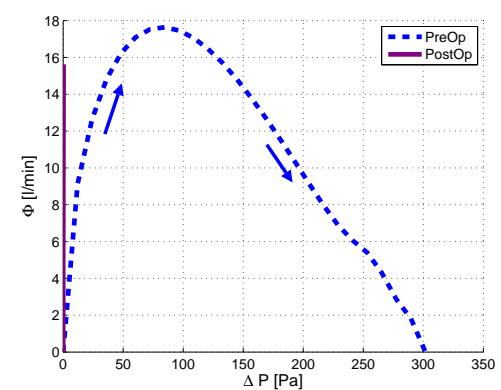

(a)

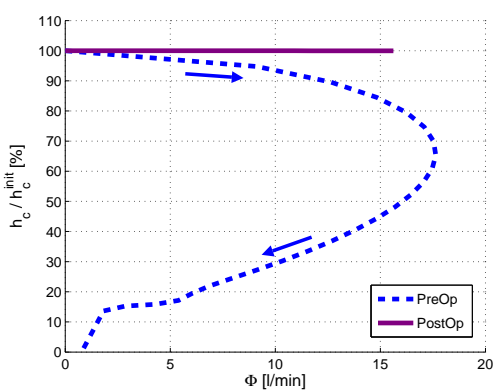

(b)

Fig. 4. Comparison between pre-operative and post-operative simulations. (a) Airflow rate $\phi$ as a function of the pressure drop $\Delta P$. (b) Constriction height $h_{c}$ as a function of $\phi$. The arrows in the pre-operative curves show the direction of increasing $\Delta P$ (from the initial configuration to the complete closure)

In a second step, a simulation has been carried out on the geometry extracted from post-operative data of the same patient. The pre-operative and post-ope- 
rative results are overlaid in figure 4 . The airflow rate $\phi$ has first been plotted as a function of the pressure drop $\Delta P$ between the inlet and the outlet of the oropharynx (figure (a)). For a typical value of $\phi$ of $10 \mathrm{l} / \mathrm{min}$, which is of the order of the rate recorded during sleep [18], it can be observed that:

- on the pre-operative simulation, the pressure drop $\Delta P$ associated to $\phi$ is approximatively $200 \mathrm{~Pa}$.

- on the post-operative simulation, the same flow rate is obtained with $\Delta P$ close to $0 \mathrm{~Pa}$ (less than $1 \mathrm{~Pa}$ ).

Indeed, the pre-operative case corresponds to a pharynx which is very narrow $(\simeq 1.5 \mathrm{~mm})$ and thus very collapsible. Therefore, it is very resistive to the transit of the airflow. Conversely, in the post-operative case, the pharynx has been enlarged up to $10.5 \mathrm{~mm}$ so that it could be approximatively considered as a straight rigid duct, which offers few resistance to the airflow. Moreover, in the pre-operative case (figure (b)), as the flow rate $\phi$ is increased, the constriction height decreases down to a value of approximatively $65 \%$ of the initial height, when $\phi$ reaches its maximum $(\simeq 17.5 \mathrm{l} / \mathrm{min})$. Then, both the constriction height and the flow rate decrease, until complete obstruction. In the post-operative case, the initial constriction height is so high that no significant decrease can be observed.

For the patient 2, the behaviour of the model is the same as for patient 1 . In the pre-operative case, the occlusive pressure ${ }^{8} P_{f}$ is respectively of $295 \mathrm{~Pa}$ and of $260 \mathrm{~Pa}$ for patients 1 and 2 . The maximal airflow $\phi_{\max }$ is approximatively $18 \mathrm{l} / \mathrm{min}(17.6 \mathrm{l} / \mathrm{min}$ for patient 1 and $18.1 \mathrm{l} / \mathrm{min}$ for patient 2$)$. For the two patients, no significant variation of the constriction height can be observed in the post-operative case.

\section{Discussion}

\subsection{Clinical Interest of the Simulation Software}

First, the two simulations from pre-operative radiographies predict an important predisposition to complete closure, while there is no more significant impact of the airflow on the upper airway deformation in the post-operative case, when the pharynx has been enlarged. This is in good agreement with clinical data (see table 1). This can be considered as a first qualitative in-vivo validation of the simulations. The second point is that the simulation time is of the order of 20 $\mathrm{mn}^{9}$. This makes the software compatible with clinical applications ${ }^{10}$. Moreover,

\footnotetext{
${ }^{8}$ the value of the outlet pressure $P_{s}$ for which complete closure occurs

${ }^{9}$ for a non-optimal version of the software, which actually is built in Matlab(TM) software optimization should lead to computation times of a few minutes

10 The time constraints due to clinical practice are difficult to estimate with precision, and might be relativized as other tasks, such as imaging or polysomnography, are
} 
with our method, it is not difficult to change the geometry from one patient to another. Indeed, a new geometry of the tongue corresponds to a new matrix $\mathbf{K}$ in the fluid-structure interaction loop (see section 2). This matrix can be easily obtained from segmentation on a radiography followed by a precomputation step. These points are important aiming at simulations for clinical studies or for examination of the effects of a given treatment (surgery or mandibular splint).

\subsection{Actual Limits and Perspectives}

The assumptions stated for these simulations could appear as very restrictive and one could wonder whether they do not prevent from taking into account important aspects of the phenomenon. However, a long work of physical analysis and of experimentation has been done in order to proove they are correct as a first approximation [8] [9] [10]. Moreover, as regarding the accessible medical data, more general assumptions and a more sophisticated formulation would be superfluous for the moment. Indeed, postoperative CT-scans or MRI, necessary for validation of a $3 \mathrm{D}$ model, are not currently available. Concerning the soft tissue, before envisaging a more complex behaviour (hyperelastic or viscoelastic), the first task would be to determine accurately their Young modulus. A first estimation has been taken from [7], but it would be better to proceed to in-vivo measurements of the tongue rheology on apneic patients, with an instrument such as the one described in [19]. This will be the first short-term perspective of this work. The second short-term perspective will be to obtain a larger sample of sagittal radiographies, so as to confirm our first results. Concerning longterm perspectives, an important one would be to extend the formulation to the tridimensional case, using CT-scans or MRI, which will of course allow to take better into account the complex morphology of the upper airway. Other sites of obstruction, such as the velopharynx or the hypopharynx [20], should be also explored. The choice of the oropharynx has been suggested by the treatment, as maxillomandibular surgery affects mostly this site. Another long-term perspective would be to build models from less restrictive assumptions, and to test through comparison with in-vitro and in-vivo measurements the impact it has on accuracy and on computation time.

\section{References}

1. Malhotra, A., White, D.: Obstructive Sleep Apnoea. The Lancet 360 (2002) 237-245

2. Young, T., Palta, M., Dempsey, J., Skatrud, J., Weber, S., Badr, S.: The Occurence of Sleep-Disordered Breathing among Middle-Aged Adults. The New England Journal Of Medicine 328(17) (1993) 1230-1235

3. Ayappa, I., Rapoport, D.: The Upper Airway in Sleep : Physiology of the Pharynx. Sleep Medicine Reviews 7(1) (2003) 9-33

very time consuming. Nevertheless, the faster the simulations run, the more comfortable it is for the clinician 
4. Li, Z., Kleinstreuer, C.: Blood Flow and Structure Interactions in a Stented Abdominal Aortic Aneurysm Model. Medical Engineering and Physics 27 (2005) 369-382

5. Tada, S., Tarbell, J.: A Computational Study of Flow in a Compliant Carotid Bifurcation-Stress Phase Angle Correlation with Shear Stress. Annals of Biomedical Engineering 33(9) (2005) 1202-1212

6. Shome, B., Wang, L., Santare, M., Prasad, A., Szeri, A., Roberts, D.: Modeling of Airflow in the Pharynx with Application to Sleep Apnea. J Biom Eng 120 (1998) 416-422

7. Malhotra, A., Huang, Y., Fogel, R., Pillar, G., Edwards, J., Kikinis, R., Loring, S., White, D.: The Male Predisposition to Pharyngeal Collapse. Am J Respir Crit Care Med 166 (2002) 1388-1395

8. Hirtum, A.V., Pelorson, X., Lagrée, P.: In Vitro Validation of Some Flow Assumptions for the Prediction of the Pressure Distribution during Obstructive Sleep Apnoea. Medical \& Biological Engineering \& Computing 43 (2005) 162-171

9. Chouly, F.: Modélisation Physique des Voies Aériennes Supérieures pour le Syndrome d'Apnées Obstructives du Sommeil. PhD thesis, Grenoble, France (2005)

10. Chouly, F., Hirtum, A.V., Lagrée, P., Pelorson, X., Payan, Y.: An Attempt to Model Fluid-Structure Interaction during Obstructive Sleep Apnea Syndrome: Numerical Simulations and Validation. Journal of Fluids and Structures (2006) submitted.

11. Payan, Y., Perrier, P.: Synthesis of V-V Sequences with a 2D Biomechanical Tongue Model Controlled by the Equilibrium Point Hypothesis. Speech Communication 22 (1997) 185-205

12. Carpenter, P., Pedley, T., eds.: Chap. 2. Flows in Deformable Tubes and Channels. Theoretical Models and Biological Applications (M. Heil and O.E. Jensen). In: Flow in Collapsible Tubes and Past Other Highly Compliant Boundaries. Kluwer (2005)

13. Napadow, V., Chen, Q., Wedeen, V., Gilbert, R.: Intramural Mechanics of the Human Tongue in Association with Physiological Deformations. J Biomech 32 (1999) 1-12

14. Zienkiewicz, O., Taylor, R.: The Finite Element Method. Basic Formulation and Linear Problems. McGraw-Hill Book Company (1989)

15. Cotin, S., Delingette, H., Ayache, N.: Real-Time Elastic Deformations of Soft Tissues for Surgery Simulation. IEEE Transactions On Visualization And Computer Graphics 5(1) (1999) 62-73

16. Lagrée, P., Lorthois, S.: The RNS/Prandtl Equations and their Link with Other Asymptotic Descriptions: Application to the Wall Shear Stress Scaling in a Constricted Pipe. International Journal of Engineering Science 43 (2005) 352-378

17. Gould, G., Whyte, K., Rhind, G., Airlie, M., Catterall, J., Shapiro, C., Douglas, N.: The Sleep Hypopnea Syndrome. Am Rev Respir Dis 137 (1988) 895-898

18. Trinder, J., Kay, A., Kleiman, J., Dunai, J.: Gender Differences in Airway Resistance during Sleep. J. Appl. Physiol. 83(6) (1997) 1986-1997

19. Bruyns, C., Ottensmeyer, M.: Measurements of Soft-Tissue Mechanical Properties to Support Development of a Physically Based Virtual Animal Model. In: MICCAI 2002. (2002) 282-289

20. Rama, A., Tekwani, S., Kushida, C.: Sites of Obstruction in Obstructive Sleep Apnea. Chest 122(4) (2002) 1139-1147 